\title{
Considering Pigeons for CARrying Delay Tolerant NetWorking BASED INTERNET TRAFFIC IN DEVELOPING COUNTRIES
}

\author{
Jeremiah Scholl \\ Health Informatics Centre \\ Karolinska Institutet \\ Sweden \\ jeremiah.scholl@ki.se
}

\author{
Anders Lindgren \\ Communication Networks and Systems Lab \\ Swedish Institute of Computer Science \\ Sweden \\ andersl@sics.se
}

\begin{abstract}
There are many regions in the developing world that suffer from poor infrastructure and lack of connection to the Internet and Public Switched Telephone Networks (PSTN). Delay Tolerant Networking (DTN) is a technology that has been advocated for providing store-andforward network connectivity in these regions over the past few years. DTN often relies on human mobility in one form or another to support transportation of DTN data. This presents a socio-technical problem related to organizing how the data should be transported. In some situations the demand for DTN traffic can exceed that which is possible to support with human mobility, so alternative mechanisms are needed. In this paper we propose using live carrier pigeons (columba livia) to transport DTN data. Carrier pigeons have been used for transporting packets of information for a long time, but have not yet been seriously considered for transporting DTN traffic. We provide arguements that this mode of DTN data transport provides promise, and should receive attention from research and development projects. We provide an overview of pigeon characteristics to analyze the feasibility of using them for data transport, and present simulations of a DTN network that utilizes pigeon transport in order to provide an initial investigation into expected performance characteristics.
\end{abstract}

\section{INTRODUCTION}

One challenge limiting the implementation of Information and Communication Technology (ICT) in developing countries is a lack of supportive infrastructure. This infrastructure can include lack of reliable and adequate power, and inadequate access to communication networks such as the Internet, and Public Switched Telephone Networks (PSTN). Even though cellulare phone connectivity is increasingly available to people in the developing world, and is providing a wide range of benefits, it is not a "one-size-fits-all" solution that solves all connectivity problems. There are for example rural regions in Africa that are not expected to be covered adequatly by cellular networks for several decades (Buys et al., 2009). There also are some ICT applications, such as transport of medical images, that have high bandwidth requirements that cannot be supported adequatly or in a cost effective way by cellular networks.

There are a number of different options that can be used to establish connectivity when the "usual" connectivty provided by large Telecom companies is inadequate however. One option is to set up point-to-point WiFi links. A Telemedicine project in south India for example has utilized this method in order to set up video conferencing based consultation between patients at clinics in rural areas where there is no Internet access, and physicians located at an urban hospital (Surana et al., 2008).

Another strategy that is being investigated is the usage of Delay Tolerant Networking (DTN). DTN is a technology that has been advocated for providing store-and-forward 
network connectivity in developing regions over the past few years (Lindgren \& Hui, 2009). There are several types of DTN networks, with one type of network being those that rely on the physical transport of data between locations. This can be accomplished in many ways. A very basic type of DTN network can rely on the transport of USB sticks that contain data between locations. Other DTNs may utilize wireless communication at local sites based on common technolgies such as WiFi or Bluetooth. A key concept with DTN is that routing protocols allow multi-hop communication to be supported. This makes it possible for two locations to be connected to each other, even if someone never travels directly between these two locations. This is possible by passing information through the DTN network, similar to a baton being passed in a relay race (Dijkstra, 1959), or by replicating and passing information, similar to an epidemic spreading (Vahdat \& Becker, 2000), or some combination of both.

DTN is still a fairly new technology and most of the research has focused on technical issues, such as routing protocols and network infrastructures (Lindgren \& Hui, 2009). A few notable projects have managed to experiment with real-world deployments of DTN systems (Pentland et al., 2002; Brewer et al., 2005; Lindgren et al., 2008). DTN has been used for example to support email and other types of communication for indigenous peoples living in Swedish national park regions (Lindgren, et al., 2008), and to support a variety of services at Internet kiosks deployed at Indian villiages (Seth et al., 2006).

Many of the socio-technical problems that need to be considered when designing sustainable DTN systems in the real world have not been widely investigated however. Since sustainability in general is a key issue affecting the overall usefulness of IT systems in developing countries (Surana et al., 2008) this is a significant issue.

One general issue that sill has not been widley investigated for example is which specific socio-technical approach should work best for transporting the data in various contexts. Today the data is generally transported together with people that are mobile in one way or another. This may be people that carry DTN-enabled devices in back-packs for example (Lindgren, et al., 2008), or that support "mechanical backhaul”, for example by driving vehicles such as DTN-enabled buses (Balasubramanian et al., 2007; Zhang et al., 2007). These strategies will work effectivly in some situations, but also can be problematic in others since "...data delivery is dependent on ... the mobility of people, no data will be delivered unless there is a constant relocation. It is also important to note that while people might be willing to carry a relay [during travel] they would normally do, it is quite unlikely that they will [travel extra], just to act as data relays” (Lindgren et al., 2008). DTN networks can thus be difficult to set up and maintain in settings where human travel is too intermittent or unreliable to support data delivery, or if the people that do travel are not willing to cooperate with the organization setting up the DTN network.

One example scenario that illustratse this problem are DTN networks that have been proposed for the amazon region based on mobility supported by boats (Coutinho et al., 2011). While this may be better than the alternative (no connectivity at all) it is a rather slow method of travel, and thus cannot support data delivery very frequently. A study of the attitudes of health workers with experience in resource poor regions also showed that while they hold quite positive views on the potential for DTN to support health work, they also feel that DTN data delivery would need to occur fairly often for these services to be useful (Syed-Abdul et al.). The aggregate response for a variety of service for example noted that $40.7 \%$ the respondants felt that data delivery would need to be once a day or more often for these services to be "useful". Organizing data delivery at short enough intervals can thus be challenging for some DTN services. 
In this paper we consider a novel approach to DTN data transport, in order to deal with the limitations that are inherant to relying on human mobilty each time data would be transported. Specifically, the concept of equipping carrier pigeons (columba livia) to support DTN data transport is presented.

The general concept of using pigeons to carry data is not new. Pigeons have been historically used for transport of small amounts of data in the form of messages written down on pieces of paper. A few years ago, they were also used (with tongue in cheek) to implement paper based transport of Internet traffic protocol (Waitzman, 1990; Ward, 2001). Although this technology did not allow the transport of "usable" levels of traffic, recent experiments have demonstrated that pigeons carrying USB sticks can deliver higher bandwidth throughput than DSL connections in Africa (Nusca, 2009).

The concept of using pigeons to fully support sustainable DTN services has however not been seriously investigated. There have been papers published on DTNs that use "homing pigeon (HoP) assisted messaging", otherwise known as "pigeon networks" (Guo et al., 2007, 2008, 2010; Zhou et al., 2010). These papers however refer to the homing pigeon as a metaphor, while describing systems that are based on the use of an aerial vehicle. The high costs and complicated technical maintainance of these vehicles may thus not make them a realistic strategy for supporting communincation in developing regions. This work aslo did not consider the practical limitations and characteristics of using real carrier pigeons to carry data, and thus cannot be used to determine the potential usefulness of DTNs that are supported by real carrier pigeons.

In this paper we investigate issues related to using real carrier pigeons to replace and/or supplement DTN data delivery that is reliant on human mobility. We argue that DTN networks based on carrier pigeon transport have the potential to offer better performance than DTN networks reliant on human mobility, and that the creation and maintainance of these networks should not hinder the sustainability of the DTN network significantly. We also provide some inital simulations of such a network in order to contribute to technical understanding of the potential performance characteristics, and engineering tradeoffs that may be inherent when engineering such networks. We hope to contribute to the development of ICT for developing countries by providing a general conceptual framework that can be used to motivate the further investigation of this solution in specific contexts, to serve specific needs.

The rest of this paper is organized as follows. In the next section an overview of relevant facts about the carrier pigeon (columba livia) are presented in order to provide a discussion of the feasibility of creating sustainable columba livia based DTNs (CoLi-DTN) in developing countries. Since this is a yet unexpored concept we follow this with a discussion of two basic architectures for CoLi-DTNs and some of the tradeoffs that they would impose. This is followed by simulations of a simple CoLi-DTN system in order to provide some basic understanding of salient issues that may affect the performance CoLi-DTN systems, and some engineering tradeoffs that may be enountered. Finally, we discuss future work in the area and conclude.

\section{FEASIBILITY OF CoLi-DTNS}

When considering the feasibility of CoLi-DTNs, a key factor is the limitations imposed by the use of actual live pigeons for carrying data. This leads to two primary considerations that need to be addressed in a real world context:

- How feasible will it be to outfit pigeons with the necessary equipment, and train them to fly "useful” routes reliably enough for the target services?

The Electronic Journal on Information Systems in Developing Countries http://www.ejisdc.org 
- How realistic is it to assume that network providers will be able to maintain a CoLiDTN in the context of developing countries?

The first question is related to the limitations that will be encountered when equipping and training carrier pigeons, and the second question is related to limitations imposed by raising and keeping them (especially in rural areas in the developing world). Each of these issues is discussed below in a separate subsection. To a large extent the analysis is based on information about pigeons that is available in the book "Microlivestock - Little-Known Small Animals with a Promising Economic Future” (Sumter, 1991).

\subsection{Equipping and Training Pigeons}

If a pigeon is going to function as a carrier of DTN data it must be able to handle a payload that is adequate for transporting the necessary DTN hardware over a "useful" route. Traditional paper based transport used scrolls attached to a pigeon's leg. This significantly limited the size and weight of the payload. In modern times additional payload containers have been developed however. Scientific programs investigating pigeon behavior (Steiner et al., 2000), and telemedicine programs transporting blood and medicines (Maubouche, 1986) have developed containers that attach the payload to the chest or back of the pigeon. With additional training these pigeons were trained to transport payloads of 35 and 39 grams respectively. As shown in Figure 1, pigeons have also been equipped with cameras used to support aerial photography. Payloads of up to 70 grams have been reported in this context. (We have not been able to confirm this first hand in an English language publication). Carrying a relativly large payload does not necessarily hinder the pigeon from flying at a fairly high rate of speed. Researchers on pigeon behavior for example have recorded pigeons equipped with 35 gram GPS devices maintaining a typical speed of around 55km/s (Steiner, et al., 2000).

Although we do not advocate a specific technical architecture in this paper (and leave this as a question for future research) the ability to carry 35-70 grams of weight does indicate that a variety of different types of CoLi-DTNs should be possible to develop. The pigeons for example could be equipped to carry USB sticks, or be equipped with special wearable computers that support for local wireless data transfer once they have landed. A discussion of the tradeoffs from each of these options is givin later in the paper.

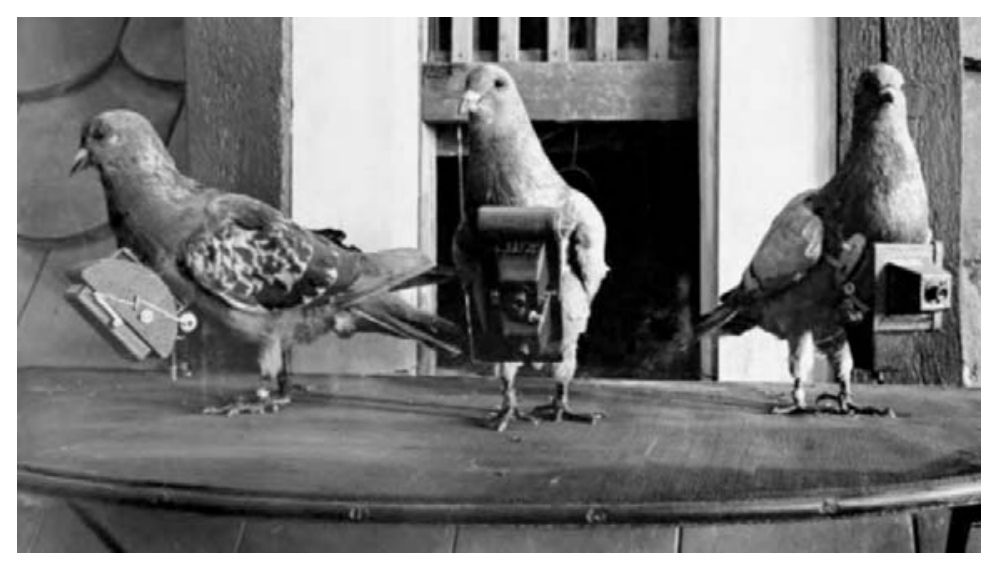

Figure 1: A photograph of pigeon photographers in the early 20th century.

In addition to equipping pigeons with relativly large payloads, modern techniques have also been developed to train pigeons to fly routes that can adequately support DTNs. 
This is an important consideration because traditional methods used for paper-based communication, and those used for performing basic experiments on transporting data with pigeons via USB stick (Nusca, 2009) impose severe restrictions as they only utilized pigeons trained to fly home to a nest at one fixed geographic location. A caged bird thus needs to be physically transported away from home to send each message. Two-way communication is difficult to set up because it requires the advance transport of caged birds with home nests at each of the two locations. Since each transported bird can only deliver one message before needing to be transported again, these training techniques require a fair degree of human mobility to keep the communication flow going.

In modern times this issue has been resolved however. Modern techniques have shown that "...pigeons can be trained to carry messages in two directions: flying from one point to another and then back again. They will do it twice a day, and with almost perfect reliability. The key is to place the feeding station at one end and the nest at the other. This limits the pigeon's range, but they still can handle round-trip distances up to $160 \mathrm{~km}$." (Sumter, 1991, p. 142). Laboratory experiments have also shown that a pigeon can be trained to go to two separate feeding stations within a small enclosure, one for morning feeding time, and one for evening feeding time (Petruso et al., 2007). The use of two feeding stations has not been verified in any practical application however, and it is still unclear if pigeons can be trained to fly reliably to two separate locations over distances that are practical for supporting a DTN.

Work has also been conducted on making the flight paths of the pigeons more flexible by training the pigeons to fly to a mobile nest that can be moved at a relatively rapid rate (Reynaud, 1898; Neubronner, 1920). This was mainly done to enable the use of pigeon communication in war-time situations when troops and command centrals are moving. While we believe this is less likely to be used in a CoLi-DTN for developing regions, it theoretically could be used to enable the use of a system like this for nomadic groups if necessary. As this work was also rather experimental and requires more labor than ordinary training of carrier pigeons, we will for the rest of the paper impose some constraints on the pigeon mobility. Each pigeon will only be trained to fly between two points, and cannot be retrained to fly routes to new locations after it has learned to fly between the nest and the feeding station. Since DTN routing allows forwarding of messages to support multi-hop routing, many-tomany DTN-based communication between locations that are not directly connected with pigeon flights will still be possible to support.

\subsection{Raising and Keeping Pigeons}

The issues in the last two subsection emphasizes how modern training, proper equipment, and the possibilities of DTN routing, allow CoLi-DTNs to support communication of much larger scale than previous systems that utilize pigeons for transport of paper or USB-sticks have allowed. The feasibility of setting up a CoLi-DTN network that supports many-to-many communication does not mean that such networks will be sustainable when used in practice however. Two additional issues need to be considered. These are economic sustainability and operational sustainability (Surana et al., 2008). The local users must be able to afford to keep the network going without needing outside donations beyond start up costs, and network maintenance needs to be conducted without needing continued outside support. This does not mean that user organizations should not need to outsource network maintenance at all, but rather that it must be possible to develop a local network manager or provider that does not need continued support from groups in developed countries that may initially help to set up the network (Surana et al., 2008). 
Inevitably realizing each of these issues in practice will be highly dependent on the local context and services in relation to the costs and benefits of the DTN. An important consideration is that supporting pigeon based transport by itself will not guarantee the sustainability of the services the network is designed to support, and we do not suggest we can solve all the related issues to provide sustainable DTN services in this paper. As explained below however, keeping and raising pigeons should not create a significant economic or operational burden in the developing context. As such, the overall sustainability of CoLi-DTNs will not be significantly hindered by the use of pigeons to carry data, and instead will be determined by other factors outside the scope of this paper, such as the the economics related to the specific services that the DTN is set up to support etc.

Carrier pigeons are a domesticated animal with breeding pairs producing from 12-14 young annually. Thus, once a breeding system is in place, it should be possible to develop a sustainable network over time through continuous breeding of new pigeons to replace lost ones, or to increase the capacity of the networks. The cost of raising the pigeons is fairly low. The last known paper based carrier pigeon delivery service in India for example was noted in 1999 to feed its pigeons for less than 5 cents a day each (Lak, 1999). Because carrier pigeons have been bred for homing purposes they also have larger than usual breast for a bird. Pigeons not needed for data transport thus can be used for meat, and at times they are bred specifically for this purpose. While climate concerns may be an issue in extreme areas (extreme arctic winters), domestic and feral pigeons live over a wide range and can be kept in a wide variety of climates including, tropical and cold winter conditions. Specific breeds have even been developed to handle desert temperatures of up to 60 degrees Celsius (Marder \& Gavrieli-Levin, 1987). In this respect we deduce that sustainable pigeon raising operations should be viable in the developing country context.

The work in keeping, training and equipping pigeons may be significant in the sense that human handlers will need to trained, and spend labor on setting up and maintaining the network. The overall labor that is necessary however can be reduced by having only a few locations in the network responsible for raising and nesting pigeons. Other locations would only need to maintain a feeding station and would generally not need to handle pigeons in order to receive or forward messages. Using this strategy it should be possible to support many-to-many communication with a "star topology" where pigeons need only to nest and be equipped at the central hub.

There will be a certain degree of human labor needed to maintain nests at a few locations however, and training pigeons to fly back and forth between the feeding station and nest can take a fair degree of persistence initially. An important consideration in some contexts is thus that CoLi-DTNs will not completely eliminate the need for human mobility between locations. Rather they will reduce the mobility to that necessary to train pigeons. Domesticated pigeons can have a 15 year life span however so there will be a fairly large reduction in necessary travel between locations when considering the total number of times the pigeons will deliver data, in comparison to the amount of mobility to train them.

In developing countries the cost of human labor is also fairly low and the training necessary to handle pigeons should not be difficult to develop locally. Similar to raising chickens, it should be possible for people with very low levels of education, including illiterate people, to handle the work. In this respect, while supporting a CoLi-DTN will introduce some labor costs, and will need to be supported by some human mobility, CoLiDTNs seem to be a potentially feasible and potentially sustainable option for DTN data delivery in situations where human mobility is highly limited, but where DTN services could provide value. 
It is thus worth investigating the technical characteristics of CoLi-DTNs in order to gain insight into the performance characteristics that might be expected from such a network if one were implemented in practice.

\subsection{Operation of CoLi-DTNs as it Relates to Different Technical Architectures}

As mentioned previously the technical implementation of a CoLi-DTN can be done in many different ways. Each of them will have different characteristics and at times they will present different operational and organizational challenges. In this section, we outline some of the tradeoffs that exist between the different types of CoLi-DTNs. Figure 2 shows two different classes and their benefits and problems.

The most basic type of CoLi-DTN worth considering is to develop high throughput and low cost solutions by equiping the pigeons with USB memory sticks that contain the data. With this type of hardware a human handler would be needed to remove the USB stick(s), download the data manually, and reattach USB stick(s) each time a pigeon lands. Such handling would be necessary at both the nests and feeding stations.

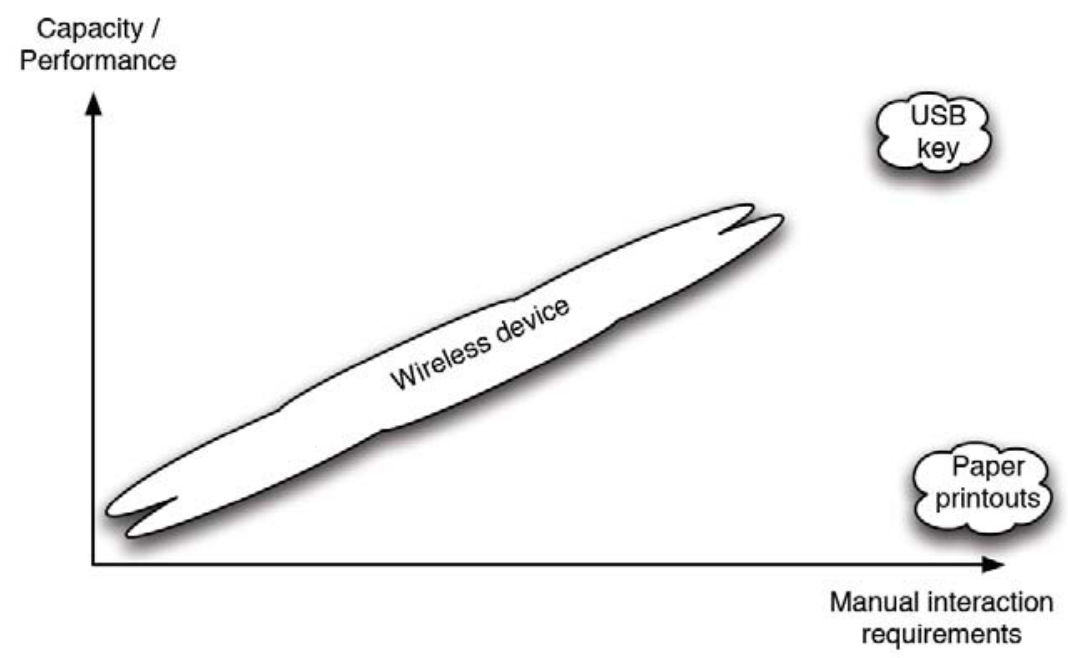

Figure 2: Different operational modes of pigeon networks.

In many contexts this should in fact be a very effective solution. The amount of handling would not be significantly different from traditional delivery methods where a human was needed to remove paper scrolls, or modern implementations where handlers have removed blood samples and medicines from containers ("Hospitals Band Together - Pigeons Flying for Life," 1977). Inductive logic thus suggests that some people interested in a pigeon based DTN would also be willing to spend the amount of labor necessary to handle the USB sticks. The amount of work can also be reduced by using technology that has been developed to support automatic closure of a pigeon cage on arrival. This would give the handler some flexibility on when to visit the feeding station or nest and perform the work.

In some situations however it may be beneficial to reduce the need for human handlers to facilitate the passing of data. It has been suggested for example to use DTN to support rural health clinics in developing countries (Scholl et al., 2009). Such clinics are often visited by a significant number of patients, are staffed by a small number of people, and interruptions in work flow can thus significantly affect the quality of care. If such a clinic were connected to a CoLi-DTN then it would benefit greatly if the feeding station only 
needed someone to visit it every once in a while in order to perform maintenance (i.e. load the feeder), rather than to handle pigeons each time one arrives.

The continuing miniaturization of hardware suggests that it should be possible to develop wearable computers that are enabled with DTN routing and local wireless connectivity (e.g., via Bluetooth, WiFi, or ZigBee), as well as batteries, in order to support this operation mode. IEEE 802.11g set ups such as the Atech iWEM-1000, which requires very little power and weighs only 5 grams, are available for example, and can provide a high throughput. Additionally, it should be possible to reduce the overall weight of the hardware by using wireless energy transfer (Chandrakasan et al., 2008). This technique has been applied to power biomedical sensors (ibid.) and also is used to power smart cards. The advantage of wireless energy transfer is that the device would be powered by an inductive field generated at the delivery points, instead of by batteries that would need to be carried by the pigeons.

There also has been a lot of additional research that may prove useful for those investigating wearable computers for pigeons. Much experience can be gathered from the field of sensor networks for wildlife habitatmonitoring (Dyo et al., 2009; Mainwaring et al., 2002; Juang et al., 2002; Sorber et al., 2007) for example. In that field, sensor and communication devices are also deployed attached to animals and have many of the same requirements we see here. Units must be small and lightweight to not impede the mobility of the animals and must also be protected from environmental factors such as rain. Much work has also been done on reducing the duty cycle of the radio (Dyo \& Mascolo, 2008) and the amount of data sent in the network (Lindgren et al., 2008) in order to reduce energy consumption and preserve battery life.

\subsection{Potential Capacity of CoLi-DTNs and Comparison to Other Technologies}

When analyzing the potential capacity for a CoLi-DTN it is interesting to compare the throughput to other networking solutions that exist in low infrastructure regions. This is because if the performance is high enough then it may be possible that CoLi-DTNs may provide value in situations where other connectivity options are available, rather than just in situations where there is no other option. Today there are a variety of connectivity options that did not exist a few years ago. Point-to-point WiFi connections for example can be delivered over long distance at a data rate of approximately $6 \mathrm{Mbps}$ of bidirectional traffic (Surana et al., 2008), and do not need to be supported by Telecom companies. It is thus interesting to analyze the potential throughput of a Coli-DTN link in comparison to point-topoint $\mathrm{WiFi}$, and other options to see if it offers competative performance.

Based on the weight limitations imposed with pigeon payloads it can be assumed that a pigeon should typically be able to carry at least 3 memory sticks of 64GB each based on the current size a weight for USB sticks. This allows for 768 GB of bidirectional traffic per day over a single link (192GB during each flight for 2 round trips each day). This works out to 71.11 Mbps of traffic, far more than the point-to-point WiFi connections and cable Internet or DSL connections in developed countries. It is worth clarifying however that the long delay for a CoLi-DTN suggests that it will not be a realistic replacement for these technologies in most cases where they are feasible to set up. Another consideration is that 64GB USB sticks are actually quite expensive at the moment, generally costing more than 100 dollars. This would not only impose significant additional costs on setting up the network, but it also would create a security risk since there would be a significant incentive for people to, for example, hunt the birds and steal the USB sticks. 
In this resepect the peformance above is not expected to be feasible for a few years. As the cost of flash memory is reduced however (as does occur over time), it is highly likley that such high thoughput should be possible in the future.

It is thus quite possible that a CoLi-DTN could be used to transport large amounts of bulk data traffic while point-to-point WiFi or other connectivity is used to provide a higher quality of service for services like audio and video conferencing. A large village for example might be able to utilize point-to-point WiFi to support all the necessary phone services, while a CoLi-DTN would be used for the remainder of Internet traffic.

\section{Simulations}

Since on of the benefits of DTN technology is that it can support multi-hop routing it is intersting to explore the characteristics of a CoLi-DTN that contains nodes that are not in direct contact. In this section, we show the results from some initial simulations performed in the OmNet++ simulator of such a system in order to theoretically explore some of the parameter space.

We evaluate the CoLi-DTN in a scenario like the one shown in Figure 3. In this scenario, pigeon routes have been designed so that the network consists of a number of "stars" - central hubs containing pigeon coops that connect nodes which only have feeding stations around them that are within flying range of a pigeon, and also links that connect these hubs to ensure that the network is fully connected. Nodes are $40-80 \mathrm{~km}$ from each other, and pigeons are expected to fly at $55 \mathrm{~km} / \mathrm{h}$ (realistic numbers based on real pigeon data). This is a likely type of network topology in order to reduce the number of pigeon nests necessary.

The simulations we present are not intended to be a complete performance study of CoLi-DTNs, but rather some initial formative evaluations to get an understanding for the potential performance of such systems. Some of the more thorough evaluations that are planned to do in the future are discussed in Section 4. In addition to investigating system performance for more general topologies, it should be possible to use simulations of this type to explore performance of specific topologies in order to get an estimate of possible performance of a real world system in a specific context before the system is implemented.

We begin by deploying a single pigeon on each edge in the graph, and we study how the network performance is affected by deploying more pigeons on the links. In these simulations, we always keep the same number of pigeons on all links, and thus do not need to consider pigeon deployment strategies. Pigeons are able to do two flights per day and when there is more than one pigeon on a link, we study both periodic and on-demand release policies for the pigeons (if there is only a single pigeon for a link, we always use periodic releases, and we always keep at least one pigeon on a periodic release schedule). Messages are sent between different edge nodes nodes in the network.

The DTN that we simulated runs a variant of Dijkstra's shortest path algorithm (Dijkstra, 1959) in the network to do the routing. If information about a destination is available at a node (calculated using Dijkstra, based on messages passed between the nodes), this information is used to route messages for that destination. Otherwise, Epidemic Routing (Vahdat \& Becker, 2000) is used so that messages for that destination is flooded in all direction to ensure speedy delivery. We study the possible message deliverable delay that can be achieved, and the potential bandwidth/goodput for different network and pigeon configurations. 


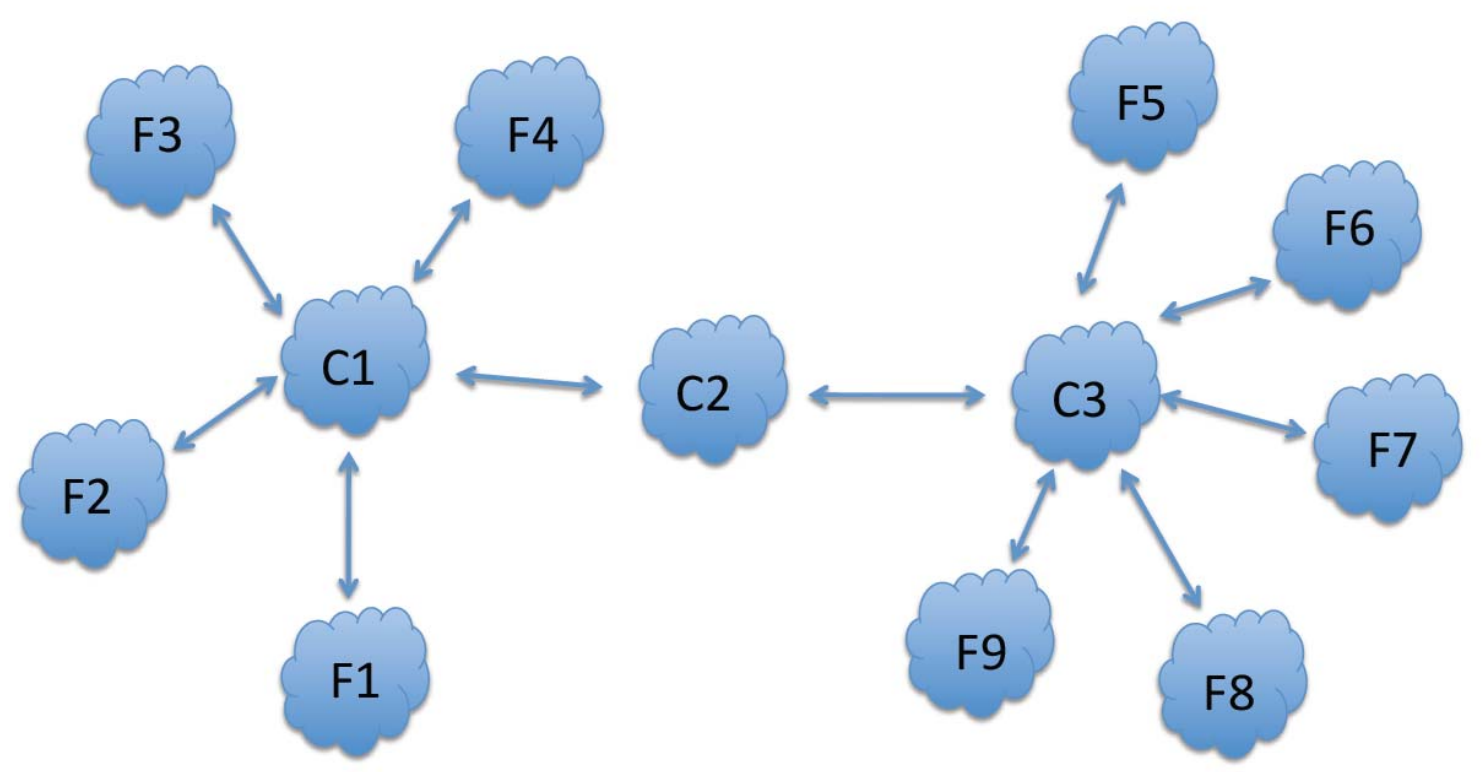

\section{Figure 3: Example of Simulation Scenario.}

\subsection{Results}

We start off by only looking at traffic generated in a subset of the topology shown in Figure 3 , namely the pigeon coop C3 and the feeding stations around that coop (F5-F9) and look at how the delay for messages sent between these nodes at the edge of the network is affected by the number of pigeons allocated to each link and the pigeon release policy used. Figure 4 shows the results from this simulation. As expected, when deploying more pigeons on each link, it is possible to reduce the end-to-end delay as less time has to be spent waiting for the departure of a new pigeon. Initially, the delay is dominated by the time spent waiting in message queues for pigeon departures, while as more pigeons are deployed, the actual propagation delay (the time it takes for the pigeons to fly between nodes) becomes the dominating factor. Therefore, we can also see that the lines are becoming flatter and it the delay cannot be reduced arbitrarily much just by deploying more pigeons. 


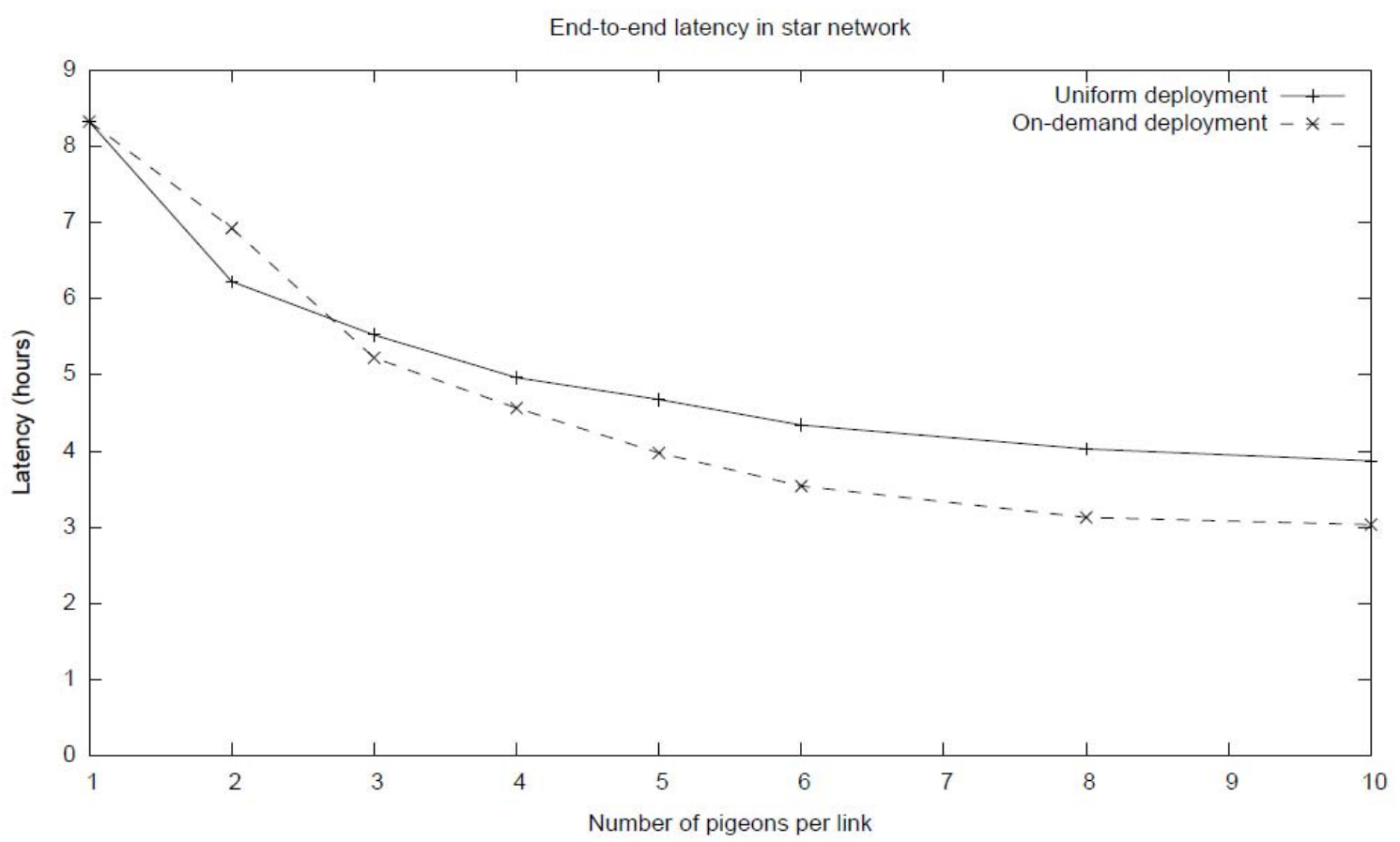

Figure 4: Average end-to-end latency for star topology. Message source and destination pairs randomly selected from nodes F5-F9.

In Figure 5, we show the same type of data, but here we are studying a data flow that goes over a larger number of hops, from node F2 to node F7. We see a similar trend in this figure as in the previous one, but the absolute latency is obviously higher here. It is interesting to note that for both situations, using an on-demand release policy actually yields worse average performance than the periodic release when only a few pigeons are available. This is likely due to pigeons being released early, causing messages arriving shortly after that to have to wait for the scheduled release, which is further away in time. This emphasizes how the performance of a Coli-DTN will not always be intuitive, and how further theoretical and concrete technical work will be needed in order to help determine the best strategies for optimal performance. As the number of available pigeons per link increases, this problem is alleviated and the benefits of the on-demand release schedule can be seen, with lower delays as a result. 


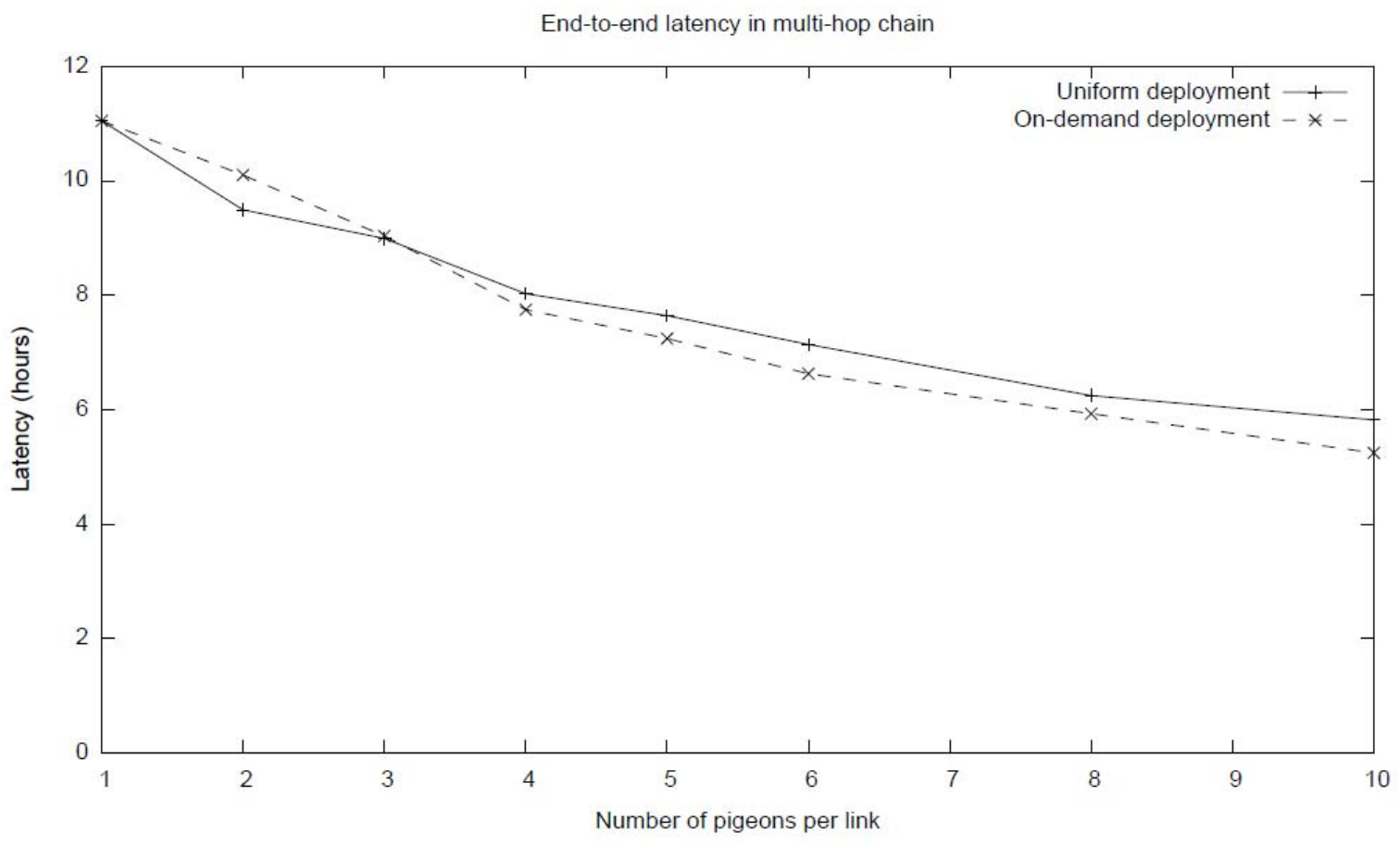

Figure 5: Average end-to-end latency for multi-hop chain topology. Data sent between nodes $F 2$ and $F 7$.

In Section 2.4, we did some analytic reasoning on the theoretical capacity of a single-hop CoLi-DTN link. We ran simulations to generate the corresponding experimental results for the multi-hop path from F2 to F7. For these simulations, we assume that data is generated at such a rate such that it is always possible to fill the complete storage space of a pigeon when it leaves the source. We also assume that a high-bandwidth solution (such as manual exchange of USB sticks) is used at the coops and feeding stations so that this transfer is not the limiting factor. Finally, we make the same assumption as in Section 2.4 that a pigeon carries 3 memory devices of 64GB each. In Figure 6, we plot the data rate that corresponds to the capacity achievable by the CoLi-DTN (i.e., the bandwidth that a direct point-to-point link would need to have in order to transfer the same amount of data over a 24 hour time period). Even with only a single pigeon available for each link, it is possible to achieve a data rate equivalent to $17 \mathrm{Mbit} / \mathrm{s}$ and as the number of pigeons increase, this also increases (at the same time as the latency decreases). It is worth noting that this simulation was only done over a single day, meaning that some data might not have reached the final destination - if the data is averaged over a longer time period, capacity would be even higher. 


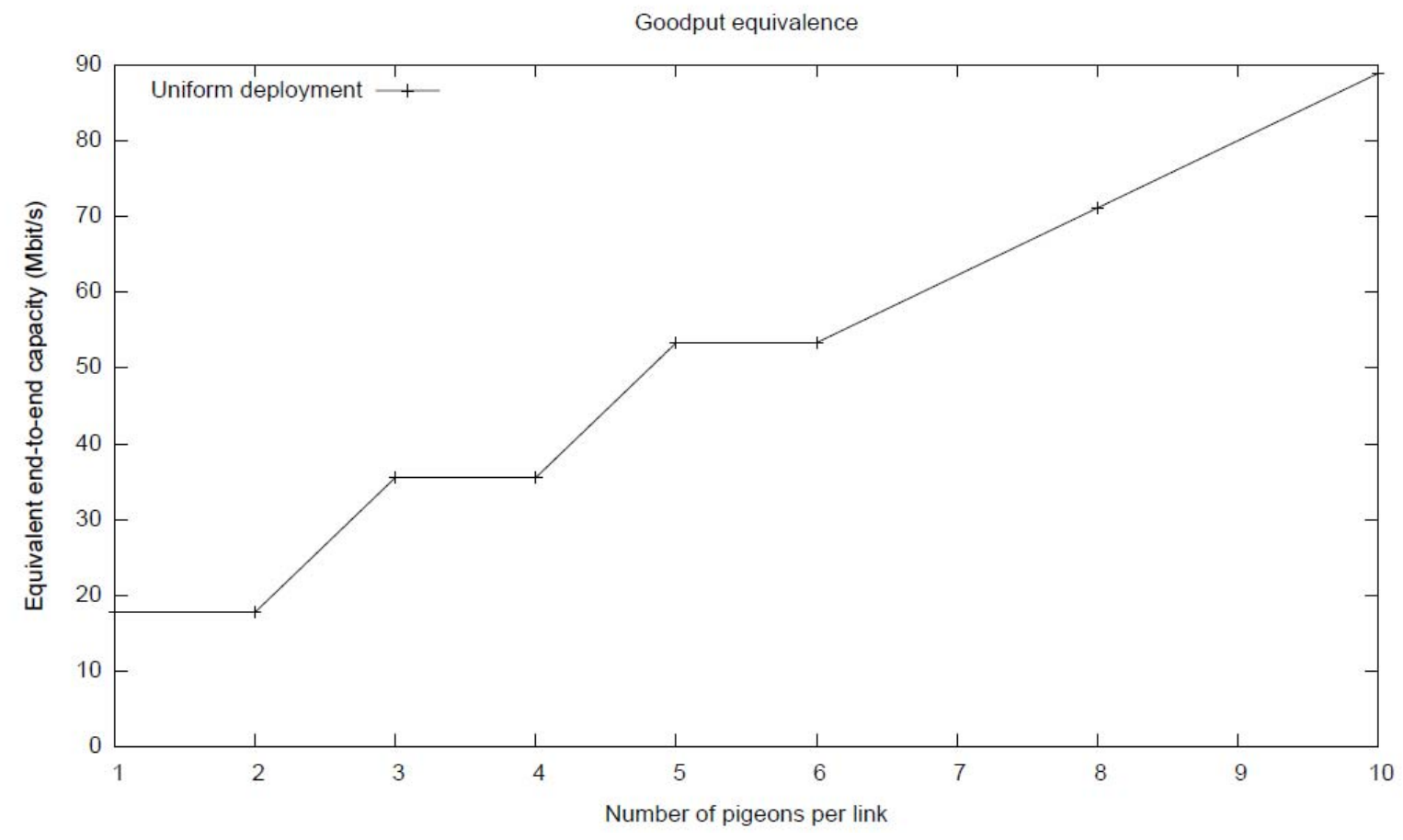

Figure 6: Capacity equivalence for multi-hop chain. Data sent between nodes F2 and F7.

\section{Discussions AND FUTURE WORK}

Pigeons have been used to transport limited amounts of data in the past. In this paper however we have argued that (with further research and development) live pigeons can also be used as a tranport mechanism to support DTNs in developing countries. We have made the following points in this regard.

- It is possible to train pigeons to fly useful routes for supporting muti-hop DTNs.

- It is possible to equipe pigeons with a large enough payload to tranport a significant amount of DTN data.

- Pigeons are easily domesticatable and inexpensive to raise in a wide range of climates.

Together these issues indicate that it should be feasible to operationally set up and maintain fairly large CoLi-DTNs in the context of developing countries. Although we have argued that CoLi-DTNs are a feasible, and potentially useful option for establishing connectivity, similar to other non-traditional connectivity options such as other DTN solutions and point-to-point WiFi, they will serve a nitch that is the most suitable option for connectivity only in select situations. There are a number of underlying contextual issues that will contribute to wheather CoLi-DTNs are the best option or not in a givin situation. These contextual issues are:

1. Existing communication infrastructure is insufficient. In many rural parts of Africa for example cellular network connectivty is not available. In other situations it might be that cellular connectivity, or other connectivity is available, but the costs are too high to justify using them for the target services. 
2. Point-to-point WiFi is not a highly suitable alternative. In some occassions for example it can be difficult to establish a line-of-sight to support point-to-point WiFi due to the presence of mountains, trees or buildings. Depending on the specifics of the implementation CoLi-DTNs may also provide better througput than point-to-point WiFi, and thus may be a better option if bandwidth demands are particularly high.

3. Travel conditions make setting up DTN delivery by mechanical backhaul or other humanbased travel insufficent. This could be the case in dense forest, mountainous regions, island areas or in areas that lack good access by roads.

As noted in the Introduction one application domain that seems quite well suited for CoLi-DTNs is support for remote health clinics. A recet survey has shown that workers with experience at these clinics are fairly positive about the possibiltiise for DTN, and also that in some situations they would prefer data delivery rather options. Another consideration is that the texts that have reported the usage of pigeons trained for two way tranport came from the medical context where these pigeons were used for transporting blood and medicines. It may thus be possible to set up CoLi-DTNs for remote clinics that enable the transport of blood and medicines in addition to the digital data.

Although CoLi-DTNs are most likley to be useful to provide connectivity while greatly reducing the amount of human mobility that is necessary to support a DTN, it is also possible that, in the future fairly high bandwidth CoLi-DTNs will be deployable. It thus is interesting to note the possibility that they may also compete with other types of Internet traffic, rather than only be useful in situations where other types of connectivity are not an option. It is quite conceivable that CoLi-DTNs will in fact be competitive against DSL, ISDN and point-to-point WiFi connections in many settings.

There are a number of issues that need to be further explored before sustainable CoLiDTNs can be set up in developing countries in a way that will provide benefit to local populations. An overview of these issues is provided in the subsections below.

\subsection{Technical Design Issues for Future Work}

One interseting issue that the the facts about pigeons presented in this paper seem to raise, is that they suggest there is a new interesting problem space within technical research that can be purused related to optimizing CoLi-DTNs.. One of the more pressing technical problems that should be addressed is the creation of functioning wearable computers for pigeons that support wireless upload and download of data with maximum efficiency and lowest cost.

Although we provided some initial simulations and analysis in order to investigate the potential peformance characteristics of CoLi-DTNs, additional work is still needed in order to gain deeper understanding of CoLi-DTNs, and to optimize network performance. Similar work has already been conducted for "pigeons" that consist of high tech flying machines (Guo et al., 2007; Zhou et al., 2010). The constraints placed by CoLi-DTNs present a new mobility model that has not been considered in current DTN research however, and also introduces new problems related to resource allocation. Two technical problems of relevance to the DTN community are allocation of new pigeons to routes, and release policies for pigeons for example. As traffic load is likely to be different on different links, an algorithm should be developed to choose which link a new pigeon should be assigned to, and trained to fly in order to optimize system performance.

There are additional factors related to the usage of live pigeons in the network that may be used to impove the problem space. Unreliable links may result in "lost” pigeons from time to time, and networks might need to be engineered for robustness in the face of 
occasional epidemics that kill a large number of the available pigeons. An additional variable that may be added to the problem space is the ability to delay the training of a pigeon to some extent after hatching. A pigeon cannot learn to fly a new route once it has been trained, and this delay may thus be useful if it is unclear which link will need a new pigeon at the time of hatching for example. Together this knowledge may be useful once CoLi-DTNs are deployed in the real world in order to advise network managers on how to get the most of the pigeons they breed.

There are also different strategies that can be investigated for pigeon release policies than we have presented in this paper. If there is only one pigeon assigned to a certain route for example, it is most likely best to allow this pigeon to do periodic flights once or twice per day. On "transit links" where much data that arrives that is the relayed by another pigeons, it might be beneficial to delay the pigeon release until incoming pigeons have arrived (within some reasonable amount of time). When more than one pigeon is available for a single link, the extra pigeons can be used to increase capacity and/or reduce the delay of the link. Reducing the delay can be done either through reducing the interval between periodic flights, or by allowing some pigeons to be released on-demand. With this second release policy, a pigeon is released as soon as some data is available for it to deliver (possibly with a short wait after that initial data arrival to allow for some more data to arrive in order to get better capacity utilization). This could greatly reduce the potential latency of the network, and given a certain number of pigeons for a link and some set of release policies, it would be possible to guarantee a certain latency for that link. This would allow the link to provide very predictable and reliable performance, which could be an advantage in some scenarios.

\subsection{Social Design Issues for Future Work}

In addition to the technical work mentioned above a number of problems related to the social design of CoLi-DTNS will need to be investigated in order to support the socio-technical design of real world CoLi-DTNs. Previous research on deploying point-to-point WiFi communication links has noted that a number of additional socio-technical problems arrise when trying to set up these communcation links for example (Surana et al., 2008). Operational suistanability in particular is challenging to achieve since local knowledge on how to set up and maintain the networks is generally not suficient before the project takes place (ibid.). It has thus been recommend that projects should focus on developing a local vendor that can run the operation of the network after the start-up phase of the project is completed (ibid.).

With CoLi-DTNs any such vendor would would need to utilize a somewhat different set of knowledge than a normal ICT vendor, as they would also need knowledge on keeping, raising and training pigeons. Although the literature has noted that others have successfully developed pigeon training methods for supporting the round trip flights that make CoLiDTNs a feasible option, there is not a significant amount of recent literature that covers the details of these modern training methods. One explanation for this is that such research has not been published because currently (before this paper) there was no clear demand for the use of two-way flights by pigeons. Instead training manuels have focused on helping people to train racing pigieons. In order to properly support any vendor organization, or other organization that will operate a CoLi-DTN some research will thus need to be conducted into how to develop these training methods and generalize them for use in a wide range of contexts.

It is also possible that in some contexts cultural factors will need to be considered when considering the best method for raising, keeping and training pigeons, especially when

The Electronic Journal on Information Systems in Developing Countries http://www.ejisdc.org 
considering that pigeons are raised for food in some developing countries. Other research on ICT for developing countries however has noted that with special care these cultural factors are possible to overcome (Brewer et al., 2006).

\section{CONCLUSIONS}

In this paper we have proposed to use columba livia birds, otherwise known as pigeons, as data mules for DTN networks. An overview of characteristics of these animals in the context of analyzing the necessary components for raising, training, and equipping them for this purpose indicates that CoLi-DTNs should be feasible to set up, and are likely to be sustainable in many contexts in developing countries. Such networks should be particularly useful in situations where DTNs could be potentially useful, but are are difficult to support due to constraints on human mobility. The amount of data that can be carried by a pigeon is large enough that it is also possible that such networks will provide better performance than other options such as wireless Internet, in many situations.

While there are many questions that will still need to be answered before people in developing countries can be expected to benefit from CoLi-DTNs, this paper suggests that this is a topic worthy of significant investigation. Future research focused on both technical and social aspects of CoLi-DTN systems has the potential to result in sustainable solutions that offer low cost connectivity to people in the developing world.

\section{ACKNOWLEDGEMENTS}

The authors would like to thank Professor Tor Sverre Lande at the University of Oslo for discussions on wireless energy transfer.

\section{REFERENCES}

Balasubramanian, A., Zhou, Y., Croft, W.B., Levine, B.N. \& Venkataramani, A. (2007) Web search from a bus. Proceedings of the Second ACM Workshop on Challenged Networks (CHANTS '07) Montreal, Canada, ACM Press, September 14.

Brewer, E., Demmer, M., Du, B., Ho, M., Kam, M., Nedevschi, S., Pal, J., Patra, R. \& Surana, S. (2005) The Case for Technology for Developing Regions. IEEE Computer, $38,6,25-38$.

Brewer, E., Michael, D., Melissa, H., Honicky, R.J. \& Surana, S. (2006) The Challenges of Technology Research for Developing Regions. IEEE Pervasive Computing, 5, 2, 1523.

Buys, P., Dasgupta, S., Thomas, T.S. \& Wheeler, D. (2009) Determinants of a Digital Divide in Sub-Saharan Africa: A Spatial Econometric Analysis of Cell Phone Coverage. World Development, 37, 9, 1494-1505.

Chandrakasan, P.A., Verma, N. \& Daly, C.D. (2008) Ultralow-Power Electronics for Biomedical Applications. Annual Review of Biomedical Engineering, 10, 247-274.

Coutinho, M., Moreira, T., Silva, E., Efrat, A. \& Johnson, T. (2011) Work-in-Progress: A New Proposal of Data Mule Network Focused on Amazon Riverine Population. Paper Presented at the Extreme Conference on Communication - The Amazon Expedition.

Dijkstra, E. (1959) A Note on Two Problems in Connexion with Graphs, Numerische Mathematik, 1, 1, 269-271.

Dyo, V., Ellwood, S.A., McDonald, D.W., Markham, A., Mascolo, C., Pásztor, B., Trigoni, N. \& Wohlers, R. (2009) Poster Abstract: Wildlife and Environmental Monitoring 
using RFID and WSN Technology, Proceedings of the 7th ACM Conference on Embedded Networked Sensor Systems. Berkeley, California, ACM Press, November 4-6.

Dyo, V. \& Mascolo, C. (2008) Efficient Node Discovery in Mobile Wireless Sensor Networks, DCOSS'08: Proceedings of the 4rd IEEE International Conference on Distributed Computing in Sensor Systems. Santorini, Greece, Springer, June 11-14.

Guo, H., Li, J. \& Qian, Y. (2008) HoP: Pigeon-Assisted Forwarding in Partitioned Wireless Networks. Proceedings of Wireless Algorithms, Systems, and Applications (WASA '08) Dallas, TX, USA, Springer, October 26-28.

Guo, H., Li, J. \& Qian, Y. (2010) HoP-DTN: Modeling and Evaluation of Homing-PigeonBased Delay-Tolerant Networks. IEEE Transactions on Vehicular Technology, 59, 2, 857-868.

Guo, H., Li, J., Washington, A.N., Liu, C., Alfred, M., Goel, R., Burge, L. \& Keiller, P. (2007) Performance Analysis of Homing Pigeon based Delay Tolerant Networks. Proceedings of IEEE Military Communications Conference (MILCOM '07) Orlando, Florida, October 29-31.

UPI (1977) Hospitals Band Together - Pigeons Flying for Life. The Milwaukee Sentinel, July $23^{\text {rd }}, 1$.

Marder, J. \& Gavrieli-Levin, I. (1987) The Heat-Acclimated Pigeon: An Ideal Physiological Model for a Desert Bird, Journal of Applied Physiology, 62, 952-958.

Juang, P., Oki, H., Wang, Y., Martonosi, M., Peh, L.-S. \& Rubenstein, D. (2002) EnergyEfficient Computing for Wildlife Tracking: Design Tradeoffs and Early Experiences with ZebraNet, Proceedings of the Tenth International Conference on Architectural Support for Programming Languages and Operating Systems (ASPLOS-X) San Jose, CA.

Lak, D. (1999) Keeping the Police Pigeons Flying BBC News Online, from http://news.bbc.co.uk/2/hi/south_asia/277047.stm

Lindgren, A., Doria, A., Lindblom, J. \& Ek, M. (2008) Networking in the Land of Northern Lights: Two Years of Experiences from DTN System Deployments. Proceedings of the ACM Workshop on Wireless Networks and Systems for Developing Regions (WiNS-DR '08) San Francisco, CA, USA, ACM Press, September 19.

Lindgren, A. \& Hui, P. (2009) The Quest for a Killer App for Opportunistic and Delay Tolerant Networks: Proceedings of the 4th ACM workshop on Challenged Networks (CHANTS '09) Bejing, China, ACM Press, September 25.

Lindgren, A., Mascolo, C., Lonegan, M. \& McConnell, B. (2008) Seal2Seal: A DelayTolerant Protocol for Contact Logging in Wildlife Monitoring Sensor Networks, Proceedings of IEEE International Conference on Mobile Ad-hoc and Sensor Systems (MASS08) Atlanta, GA, USA IEEE, September 29 - October 2.

Mainwaring, A., Culler, D., Polastre, J., Szewczyk, R. \& Anderson, J. (2002) Wireless Sensor Networks for Habitat Monitoring, Proceedings of the 1st ACM international Workshop on Wireless Sensor Networks and Applications (WSNA '02) Atlanta, GA, USA, ACM Press, September 28.

Maubouche, S. (1986) Frontiers of Medicine: On a Wing And a Test Tube The Washington Post, December $2^{\text {nd }} ., 1$. 
Neubronner, J. (1920) 55 Jahre Liebhaberphotograph: Erinnerungen mitgeteilt bei Gelegenheit des fünfzehnjährigen Bestehens der Fabrik für Trockenklebematerial (in German): Frankfurt am Main: Gebrüder Knauer.

Nusca, A. (2009) In South Africa, Carrier Pigeon Faster than Broadband, BBC News Online http://news.bbc.co.uk/2/hi/8248056.stm

Pentland, A., Fletcher, R. \& Hasson, A.A. (2002) A Road to Universal Broadband Connectivity, Proceedings of the 2nd International Conference on Open Collaborative Design for Sustainable Innovation, Bangalore, India.

Petruso, E.J., Fuchs, T. \& Bingman, V.P. (2007) Time-Space Learning in Homing Pigeons. Animal Cognition, 10, 2, 181-188

Reynaud, G. (1898) Les Lois de l'Orientation Chez les Animaux, Revue des Deux Mondes, 146, 380-402.

Scholl, J., Lambrinos, L. \& Lindgren, A. (2009) Rural Telemedicine Networks Using Storeand-Forward Voice-over-IP, Proceedings of MIE 2009, The XXIInd International Congress of the European Federation for Medical Informatics, Sarajevo, Bosnia and Herzegovina, IOS Press, August 30 - September 2, 448-452.

Seth, A., Kroeker, D., Zaharia, M., Guo, S. \& Keshav, S. (2006) Low-Cost Communication for Rural Internet Kiosks Using Mechanical Backhaul, Proceedings of the 12th Annual International Conference on Mobile Computing and Networking, Los Angeles, CA, USA, ACM Press, September 24-29, 334-345.

Sorber, J., Kostadinov, A., Garber, M., Brennan, M., Corner, M.D. \& Berger, E.D. (2007) Eon: A Language and Runtime System for Perpetual Systems, Proceedings of the Fifth International ACM Conference on Embedded Networked Sensor Systems, Sydney, Australia.

Steiner, I., Burgi, C., Werffeli, S., Dell'omo, G., Vale nti, P., Troster, G., Wolfera, D.P. \& Lipp, H-P. (2000) A GPS Logger and Software for Analysis of Homing in Pigeons and Small Mammals. Physiology \& Behavior, 71, 589-596.

Sumter, S.C. (1991) Microlivestock - Little Known Small Animals With a Promising Economic Future: National Academy Press, Washington, D.C., USA

Surana, S., Patra, R., Nedevschi, S. \& Brewer, E. (2008) Deploying a Rural Wireless Telemedicine System: Experiences in Sustainability. IEEE Computer, 41, 6, 48-56.

Surana, S., Patra, R., Nedevschi, S., Ramos, M., Subramanian, L., Ben-David, Y. \& Brewer, E. (2008) Beyond Pilots: Keeping Rural Wireless Networks Alive, Proceedings of USENIX Symposium on Networked Systems Design \& Implementation, San Francisco, CA, USA, April 16-18.

Syed-Abdul, S., Scholl, J., Lee, P., Jian, W.-S., Liou, D.-M. \& Li, Y.-C. (2012) Study on the Potential for Delay Tolerant Networks by Health Workers in Low Resource Settings. Computer Methods and Programs in Biomedicine, 107, 3, 557-564

Vahdat, A. \& Becker, D. (2000) Epidemic Routing for Partially Connected Ad Hoc Networks., Technical Report CS-200006, Department of Computer Science. Duke University, Durham, NC, USA

Waitzman, D. (1990) IETF RFC 1149: A Standard for the Transmission of IP Datagrams on Avian Carriers. http://www.ietf.org/rfc/rfc1149.txt

Ward, M. (2001) The Net Takes to the Air: 
http://news.bbc.co.uk/2/hi/science/nature/1321176.stm

Zhang, X., Kurose, J., Levine, B.N., Towsley, D. \& Zhang, H. (2007) Study of a Bus-Based Disruption-Tolerant Network: Mobility Modeling and Impact on Routing. Proceedings of ACM International Conference on Mobile Computing and Networking, Montreal, Canada, ACM Press, September 9-14.

Zhou, J., Li, J. \& Burge, L. (2010) Efficient Scheduling of Pigeons for a Constrained Delay Tolerant Application. EURASIP Journal on Wireless Communications and Networking - Special issue on wireless network algorithms, systems, and applications, 1-7: http://jwcn.eurasipjournals.com/content/pdf/1687-1499-2010-142921.pdf. 